\section{Broth cultures yield vs traditional approach in the workup of infectious keratitis}

\begin{abstract}
Purpose To elucidate whether BACTEC Peds Plus F broth, usually used for culturing body fluids in paediatric departments, can be used for corneal cultures from cases with clinically suspected infectious keratitis, and to compare yields between this method and traditional methods (blood agar, chocolate agar, a fungal media, and swab transport media).

Methods All cases with newly diagnosed, nonviral, clinically suspected infectious keratitis with no prior antibiotic therapy, were cultured both in the BACTEC Peds Plus F broth and the traditional method. McNemar's test was used for pairwise comparisons of the rates of positive growth between the two groups.
\end{abstract}

Results In total, 30 eyes were included in this study. The growth rates for the traditional method and the BACTEC broth were similar (50.0 and $53.33 \%$, respectively, $P=1.0$ ). The overall growth rate for the two methods combined was $73.33 \%$, which is $45.29 \%$ higher than the reported yield in the literature (average of $50.47 \%$ ).

Conclusions Our results show that BACTEC Peds Plus F broth can be used successfully in the work-up of clinically suspected infectious keratitis. The method has, apparently, several advantages over the 'Traditional method:' time-savings, as only one medium needs to be inoculated, transportation to the laboratory is simpler as there is no need for immediate incubation, and there is no need to keep and maintain a supply of fresh agar media. This method is especially suitable for office settings and remote clinics, but also can be used in hospital setting, as an adjunct, when available, to increase the growth yield.

Eye (2006) 20, 215-220. doi:10.1038/sj.eye.6701858; published online 18 March 2005
A Kratz, J Levy, I Klemperer and T Lifshitz

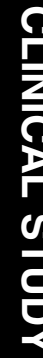

Keywords: keratitis; infectious; BACTEC; culture; yield

\section{Introduction}

Infectious keratitis is a leading cause of monocular blindness worldwide. ${ }^{1}$ Furthermore, in developing countries, it has been recognized by the WHO as a 'silent epidemic' with an incidence rate up to 113 per $100000(0.11 \%){ }^{2}$ Corneal blindness is the end result in the majority of these infections, and more disastrous outcomes such as corneal perforation, endophthalmitis, or phthisis may occur.

Extensive literature describes a standard technique for evaluating clinically suspected infectious keratitis, which includes obtaining corneal scrapings for smears and cultures prior to initiating antibiotic treatment. ${ }^{3-4}$ The traditional evaluation of clinically suspected infectious keratitis includes Gram stains, as well as multiple cultures. Culture media commonly used include blood agar, chocolate agar, and a fungal medium such as Sabouraud agar. ${ }^{5}$ Nevertheless, it was reported in Southern California that as many as $48 \%$ of patients with clinically suspected infectious keratitis received antibiotic therapy without prior culture, and only $22 \%$ of the general ophthalmologists were found to maintain culture media supplies in their offices. ${ }^{6}$

Several reasons have been offered as explanations for the disparity between the traditionally recommended evaluation of infectious keratitis and actual practice. These explanations include laboratory costs, time constraints in busy office settings, the cost of maintaining fresh media in an office setting, and the difficulty of transporting the inoculated plates, as they require immediate transportation
Department of

Ophthalmology,

Soroka University Medical Center, Faculty of Health

Sciences, Ben-Gurion University of the Negev, Beer-Sheva, Israel

Correspondence: Assaf Kratz, Department of Ophthalmology, Soroka University Medical Center, PO Box 151,

Beer-Sheva 84101, Israel Tel: + 972523443017 ; Fax: + 97286519941 .

E-mail: assaff@

bgumail.bgu.ac.il

Received: 17 August 2004 Accepted: 5 January 2005 Published online: 18 March 2005

The authors have no proprietary interest in any of the materials or techniques used in this study 
in special conditions. Other reasons can be the sense that empiric treatment is adequate for the majority of cases and a past experience of low yield from the cultures.

Contrary to the traditional culture media, swab transport media are potentially less expensive and simpler to maintain, inoculate, and transport. It has also been reported that, in certain conditions, transport media can yield as high a growth percentage of bacteria as blood agar plates. ${ }^{7}$ Unfortunately, the yield of the transport medium is dramatically reduced with time from the primary inoculation.

BACTEC Peds Plus F (Becton Dickinson, Sparks, MD, USA) (Figure 1) is a specialized broth medium that accommodates small-volume samples $(<3 \mathrm{ml})$. The medium is an enriched soybean-casein digest broth, and contains resins for antibiotic neutralization. The BACTEC Peds Plus F is a well-known and widely used broth culture medium for blood and other body fluids in paediatric practice. It is suitable for aerobic and anaerobic bacteria as well as fungi. ${ }^{8}$ These characteristics of the BACTEC Peds Plus F broth, along with no need for immediate incubation and easy transportation, makes it potentially advantageous as the swab transport medium in ophthalmologic settings.

A literature search revealed that the use of commercial broth media in the work-up of infectious keratitis has not been reported previously. The primary goal of this study was to elucidate whether the BACTEC Peds Plus F could be used for corneal cultures from clinically suspected infectious keratitis. Secondary goals were to compare the yield between this method and the traditional methods, and to review the relevant literature.

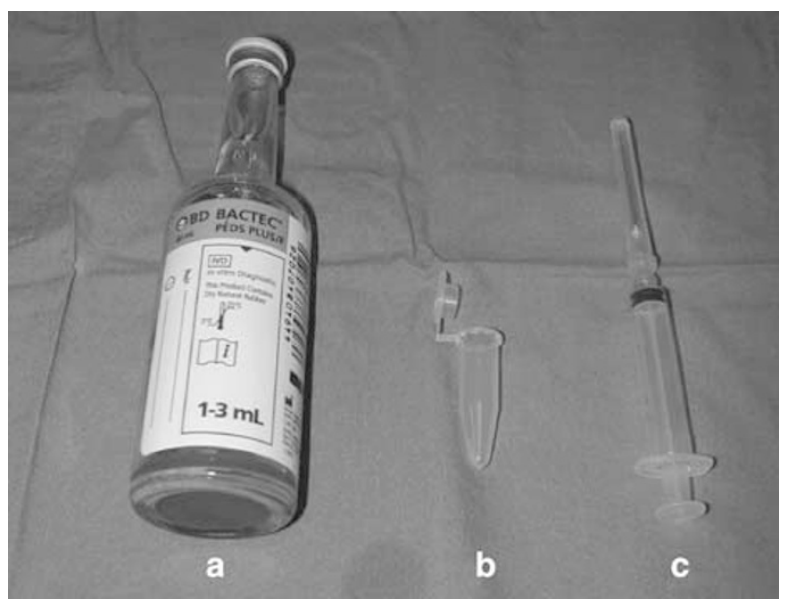

Figure 1 (a) BACTEC Peds Plus F (Becton Dickinson, Sparks, MD, USA), (b) Eppi 40 tube (Eppendorf International), (c) Regular $2.5 \mathrm{ml}$ syringe.

\section{Methods}

All cases with newly diagnosed, nonviral, clinically suspected infectious keratitis presented at Soroka University Medical Center, Beer-Sheva, Israel, between June 2003 and June 2004 were included in this prospective, comparative study. Cases with any prior antibiotic treatment, or a clinically suspected viral keratitis, were excluded. Corneal scrapings were obtained by a single ophthalmologist (AK) from all patients complying with the above criteria. A common protocol allowing the diagnosis of bacterial and fungal keratitis was used in all cases. Under topical anaesthesia (Benoxinate $\mathrm{HCl} 0.4 \%$ ) and slit-lamp magnification, corneal scrapings were obtained from the base and edge of the ulcer using three different sterile surgical blades (no. 15). The samples were inoculated directly onto blood agar, chocolate agar, and Sabouraud agar. Another scraping was carried out, using a different blade, and the specimens were inserted to a regular sterile Eppi 40 tube (Eppendorf International) previously filled with $1.5 \mathrm{ml}$ of sterile saline $0.9 \%$. The fluid from the Eppi 40 tube was then drawn using a regular $2.5 \mathrm{ml}$ syringe, and inserted into regular BACTEC Peds Plus F broth (Becton Dickinson, Sparks, MD, USA) (Figure 1). For each case, an additional sample was taken, using a regular amine agar gel transport swab without charcoal (COPAN Venturi Transystem) for secondary inoculation at the bacteriology laboratory in our medical centre. There was no policy regarding the order in which these two scrapings and swab were taken. The blood and chocolate agar plates were incubated at $37^{\circ} \mathrm{C}$. Blood agar plates were incubated under both aerobic and anaerobic conditions, chocolate agar plates were incubated in 5\% carbon dioxide, and Sabouraud agar plates were incubated at $25^{\circ} \mathrm{C}$ to enhance the growth of fungi. The BACTEC broths were evaluated in the bacteriology department in our medical centre using the BACTEC 9240 system (Becton Dickinson Diagnostic Instrument Systems, Towson, MD, USA). The blood and chocolate agar plates, the BACTEC broths and the Sabouraud agar plates, were incubated for 3, 7, and 14 days, respectively, before being reported as 'no growth.' Any isolation of a definitive, noncontaminant keratitiscausing pathogen was defined as 'positive growth.' For each case, the growth outcomes of the blood, chocolate, and Sabouraud agar were summed and were called the 'Traditional method.' These results were added to the secondary growth from the swab, and were compared with the growth outcome from the BACTEC alone. McNemar's test was used for pairwise comparisons of the rates of positive growth between the two groups. $P<0.05$ was used to denote a significant statistical difference. 


\section{Results}

In total, 30 eyes (17 right and 13 left) of 30 patients (19 males and 11 females) with clinically suspected infectious keratitis were included in this study. The growth results in the BACTEC group and the "Traditional method' + swab transport media group is shown in Table 1.

The yield of the two culture methods is outlined in Figure 2. The BACTEC method yielded $53.33 \%$ growth (16 cases), while the yield in the Traditional method + swab transport media group was $50.0 \%$ (15 cases). There was no statistically significant difference between the two groups $\left(P=1.0, \chi^{2}=0.0\right)$. As can be seen in Table 1, in some cases both methods were able to identify an organism, while in other cases only one method succeeded, or even both methods failed.

The combined positive growth of both BACTEC and Traditional Method + swab transport media is $73.33 \%$ (22/30 cases), and is statistically significant higher than the yield of the Traditional method + swab transport medium alone $\left(P=0.024, \chi^{2}=5.14\right)$, and also statistically significant higher than the yield of the BACTEC alone $\left(P=0.042, \chi^{2}=4.17\right)$.
The type and rate of identified organisms is outlined in Table 2. The most common identified organism in our series was coagulase-negative Staphylococcus, which was discovered in $50.0 \%(11 / 22)$ of all positive identifications.

\section{Discussion}

The traditional academic approach for the initial diagnosis of clinically suspected infectious keratitis

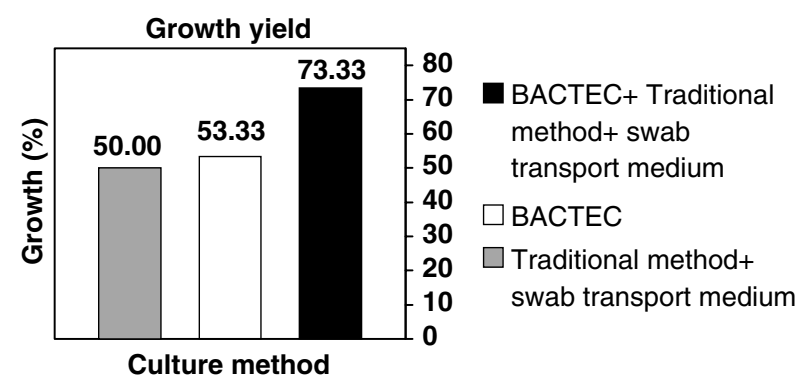

Figure 2 The distribution of growth results in the Traditional method + swab transport medium group and the BACTEC group.

Table 1 The growth outcome of the culture methods

\begin{tabular}{|c|c|c|}
\hline Patient number & BACTEC broth & Traditional method + swab transport medium \\
\hline 1 & Coagulase-negative Staphylococcus & - \\
\hline 2 & Enterobacter & Enterobacter \\
\hline 3 & - & Staphylococcus aureus \\
\hline 4 & Coagulase-negative Staphylococcus & Coagulase-negative Staphylococcus \\
\hline 5 & - & Coagulase-negative Staphylococcus \\
\hline 6 & Escherichia coli & - \\
\hline 7 & Coagulase-negative Staphylococcus & - \\
\hline 8 & - & - \\
\hline 9 & - & Enterobacter \\
\hline 10 & Coagulase-negative Staphylococcus & - \\
\hline 11 & Coagulase-negative Staphylococcus & - \\
\hline 12 & - & - \\
\hline 13 & - & - \\
\hline 14 & Candida parapsilosis & Candida parapsilosis \\
\hline 15 & Coagulase-negative Staphylococcus & - \\
\hline 16 & Moraxella catarrhalis & Moraxella catarrhalis \\
\hline 17 & Coagulase-negative Staphylococcus & Coagulase-negative Staphylococcus \\
\hline 18 & - & - \\
\hline 19 & - & - \\
\hline 20 & - & - \\
\hline 21 & Streptococcus pneumonia & Streptococcus pneumonia \\
\hline 22 & - & Coagulase-negative Staphylococcus \\
\hline 23 & - & Streptococcus pneumonia \\
\hline 24 & Coagulase-negative Staphylococcus & Coagulase-negative Staphylococcus \\
\hline 25 & - & - \\
\hline 26 & - & Coagulase-negative Staphylococcus \\
\hline 27 & Streptococcus pneumonia & Streptococcus pneumonia \\
\hline 28 & Acinetobacter & - \\
\hline 29 & - & - \\
\hline 30 & Streptococcus pneumonia & Streptococcus pneumonia \\
\hline
\end{tabular}


Table 2 Type and number (\%) of identified organisms from positive cases

\begin{tabular}{lccc}
\hline Organism & BACTEC $(\mathrm{n}=16)$ & $\begin{array}{c}\text { Traditional method }+ \text { swab } \\
\text { transport medium }(\mathrm{n}=15)\end{array}$ & $\begin{array}{c}\text { Total }(\mathrm{n}=22) \\
\text { Coagulase-negative Staphylococcus }\end{array}$ \\
Streptococcus pneumonia & $8(50.0 \%)$ & $6(40.0 \%)$ & $11(50.0 \%)$ \\
Enterobacter & $3(18.75 \%)$ & $4(26.67 \%)$ & $4(18.18 \%)$ \\
Staphylococcus aureus & $1(6.25 \%)$ & $2(13.33 \%)$ & $2(9.09 \%)$ \\
Escherichia coli & $0(0 \%)$ & $1(6.67 \%)$ & $1(4.54 \%)$ \\
Acinetobacter & $1(6.25 \%)$ & $0(0 \%)$ & $1(4.54 \%)$ \\
Moraxella catarrhalis & $1(6.25 \%)$ & $0(0 \%)$ & $1(4.54 \%)$ \\
Candida parapsilosis & $1(6.25 \%)$ & $1(6.67 \%)$ & $1(4.54 \%)$ \\
\hline
\end{tabular}

considers diagnostic scraping of corneal ulcers mandatory. ${ }^{5,9}$ In most cases, standard media to be inoculated include blood and chocolate agar for recovery of aerobic and facultatively anaerobic bacteria and fungi, and Sabouraud agar for recovery of fungi. ${ }^{5}$ Nevertheless, several recent reports describe a decrease in the frequency with which ophthalmologists perform standard cultures, and an increasing use of transport media for submitting samples to microbiology laboratories for evaluation. ${ }^{6,10,11}$

The disparity between traditional academic and actual practice can be explained by laboratory costs, time constraints in busy office settings, the cost in maintaining fresh media in an office setting, and the difficulty in transporting the inoculated plates, as they require immediate transportation in a special conditions. Another reason can be the sense that empiric treatment is adequate for the majority of cases.

Unfortunately, even when standard cultures were obtained, the growth yield were disappointing. It is interesting that even with direct inoculation of a known organism (Streptococcus pneumonia) onto rabbit cornea, ${ }^{7}$ the growth yield of the blood and chocolate agar reached only $69 \%$. Table 3 lists several reports of infectious keratitis studies in humans, in which the data concerning the growth yield were represented or could be calculated. A summary of 7888 cases reported in the literature reveals that the average growth yield of the Traditional culture medium is only $50.47 \%$. The yield of the Traditional method + swab transport medium in our study $(50.0 \%)$ is similar to the average value found in the literature, but when pooling the results from the Traditional + swab transport medium with the yield of the BACTEC in our study, the outcome is a high rate of $73.33 \%$.

The use of a special broth-based culture medium to improve the growth yield in infectious keratitis was previously described in two reports (Table 4). ${ }^{18,19}$ During a 5-year study period, Simcock et $a l^{18}$ reported that the use of a broth increased the growth yield from an average of $41.5 \%$ (81 of 195 cases) when using Traditional
Table 3 Reported growth yield of the Traditional method

\begin{tabular}{|c|c|c|c|c|}
\hline Author & $\begin{array}{l}\text { No. of } \\
\text { cases }\end{array}$ & $\begin{array}{l}\text { Growth } \\
\text { yield } \\
(\%)\end{array}$ & Type of study & $\begin{array}{l}\text { Time } \\
\text { period }\end{array}$ \\
\hline Forster $^{11}$ & 5845 & 49.0 & Retrospective & 28 years \\
\hline Sharma et $a l^{12}$ & 1088 & 53.5 & Prospective & 8 years \\
\hline Wahl et $a l^{13}$ & 130 & 40.0 & Retrospective & 5 years \\
\hline $\operatorname{Dart}^{14}$ & 53 & 60.4 & Prospective & 10 months \\
\hline Liesegang et $a l^{15}$ & 663 & 56.0 & Retrospective & 9 years \\
\hline Waxman et al $^{16}$ & 35 & 68.6 & Retrospective & 1 year \\
\hline McLeod et $a l^{17}$ & 74 & 75.7 & Retrospective & 2.5 years \\
\hline Total & 7888 & 50.47 & & \\
\hline \multirow[t]{3}{*}{ Our study } & 30 & 50.0 & $\begin{array}{l}\text { Traditional }+ \text { swab } \\
\text { transport medium }\end{array}$ & \\
\hline & & 53.33 & BACTEC & \\
\hline & & 73.33 & Combined & \\
\hline
\end{tabular}

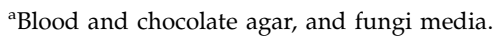

methods alone, to $79.4 \%$ (54 of 68 cases) when adding the use of the broth. Schonheyder et al ${ }^{19}$ found a yield of $85.2 \%$ when using a broth in a 1-year study (23 of 27 cases), but they did not report the yield in cases without broth use. In both studies, the broths were prepared locally in the same institute in which the studies were conducted, and were not available commercially.

As seen in Table 2, the most commonly identified organism in our study, both in the traditional method and BACTEC broth, was coagulase-negative Staphylococcus. In the past, coagulase-negative Staphylococcus was often disregarded as a significant cause of keratitis because of its presence on the skin and eyelids as normal flora. ${ }^{20,21}$ However, it is now clear that coagulase-negative Staphylococcus is an important causative organism in infectious keratitis, as shown in many studies worldwide, ${ }^{12,13,16,19,22,23}$ as well as in another study from our country. ${ }^{24}$ Our results are consistent with these findings. Measures should be taken to decrease the probability of contaminations during specimen collection. These measures include preventing 
the patient from blinking during corneal scraping and disinfecting the broth rubber head with $70 \%$ alcohol (or equivalent solution) prior to specimen injection.

The BACTEC Peds Plus F is a well-known and widely used broth culture medium for blood and other body fluids in paediatric practice. It is suitable for aerobic and anaerobic bacteria, as well as fungi, ${ }^{8}$ as proven by the growth of Candida parapsilosis in one of our cases.

The BACTEC method has previously been shown to be superior to Traditional methods when culturing blood and synovial fluids. ${ }^{25,26}$ Moreover, its sensitivity relative to the Traditional method led to the recognition of formerly rare pathogens as relatively common causative pathogens in various paediatric diseases. ${ }^{27,28}$

The approximate cost (in US\$) of both methods, as was collected from several on-line sources, is $\sim 7.5 \$$ for the traditional method and $\sim 10 \$$ for the BACTEC broth.

Our results show the efficacy of the BACTEC broth in the workup of clinically suspected infectious keratitis. When using BACTEC along with the Traditional method + swab transport medium, there is a statistically significant higher yield than using either methods alone. Adding this to the other advantages of this method, namely a single culture medium, time-savings, easy transportation, no need to keep and maintain a supply of fresh agar media, and no need for immediate incubation, the BACTEC broth, though slightly more expensive, is a worthy adjunct to the Traditional method + swab transport media in the workup of clinically suspected infectious keratitis. This method is especially suitable for office settings and remote clinics, but also can be used in hospital setting, as an adjunct, when available, as it will probably increase the total growth yield.

\section{References}

1 Whitcher JP, Srinivasan M, Upadhyay MP. Corneal blindness: a global perspective. Bull World Health Organ 2001; 79(3): 214-221.

2 Whitcher JP, Srinivasan M. Corneal ulceration in the developing world - a silent epidemic. Br J Ophthalmol 1997; 81: 622-623.

3 American Academy of Ophthalmology. Basic and Clinical Science Course, Sect. 8. External Disease and Cornea. American Academy of Ophthalmology: San Francisco, 1992, p 61.

4 Cullom RD, Chang B. Diagnosis and Treatment of Eye Disease, 2nd edn. JB Lippincort: Philadelphia, 1994, p 70.

5 Jones DB, Leisegang TJ, Robinson NM. Laboratory Diagnosis of Ocular Infections, Cumitech 13. American Society for Microbiology: Washington, DC, 1981.

6 McDonnell PJ, Nobe J, Gauderman WJ, Lee P, Aiello A, Trousdale M. Community care of corneal ulcers. Am J Ophthalmol. 1992; 114(5): 531-538.

7 Kuo IC, Cevallos V, Troyer R, Lietman TM, McLeod SD. Efficacy of transport media use versus direct inoculation of blood agar plates in the microbiologic evaluation of 
experimental Streptococcus pneumoniae keratitis. Cornea 2003 22(3): 249-253.

8 Welby PL, Zusag TM, Storch GA. Comparison of the BACTEC Peds Plus pediatric blood culture vial with Roche pediatric Septi-Chek for blood cultures from pediatric patients. J Clin Microbiol 1992; 30(5): 1361-1362.

9 Matoba AY. Infectious keratitis. In: Garratt S (ed). Focal Points: Clinical Modules for Ophthalmologists. American Academy of Ophthalmology: San Francisco, 1992, pp 8-10.

10 McLeod SD, DeBacker CM, Viana MA. Differential care of corneal ulcers in the community based on apparent severity. Ophthalmology 1996; 103(3): 479-484.

11 Forster RK. Conrad Berens Lecture. The management of infectious keratitis as we approach the 21st century. CLAO J 1998; 24(3): 175-180.

12 Sharma S, Kunimoto DY, Gopinathan U, Athmanathan S, Garg P, Rao GN. Evaluation of corneal scraping smear examination methods in the diagnosis of bacterial and fungal keratitis: a survey of eight years of laboratory experience. Cornea 2002; 21(7): 643-647.

13 Wahl JC, Katz HR, Abrams DA. Infectious keratitis in Baltimore. Ann Ophthalmol. 1991; 23(6): 234-237.

14 Dart JK. Predisposing factors in microbial keratitis: the significance of contact lens wear. Br J Ophthalmol 1988; 72(12): 926-930.

15 Liesegang TJ, Forster RK. Spectrum of microbial keratitis in South Florida. Am J Ophthalmol 1980; 90(1): 38-47.

16 Waxman E, Chechelnitsky M, Mannis MJ, Schwab IR. Single culture media in infectious keratitis. Cornea 1999; 18(3): 257-261.

17 McLeod SD, Kolahdouz-Isfahani A, Rostamian K, Flowers CW, Lee PP, McDonnell PJ. The role of smears, cultures, and antibiotic sensitivity testing in the management of suspected infectious keratitis. Ophthalmology 1996; 103(1): 23-28.
18 Simcock PR, Butcher JM, Armstrong M, Lloyd IC, Tullo AB. Investigation of microbial keratitis: an audit from 1988-1992. Acta Ophthalmol Scand 1996; 74(2): 183-186.

19 Schonheyder HC, Pedersen JK, Naeser K. Experience with a broth culture technique for diagnosis of bacterial keratitis. Acta Ophthalmol Scand 1997; 75(5): 592-594.

20 Jones DB. Early diagnosis and therapy of bacterial corneal ulcers. Int Ophthalmol Clin 1973; 13(4): 1-29.

21 Smolin G, Okumoto M, Nozik RA. The microbial flora in extended-wear soft contact-lens wearers. Am J Ophthalmol 1979; 88(3 Part 2): 543-547.

22 Benson WH, Lanier JD. Comparison of techniques for culturing corneal ulcers. Ophthalmology 1992; 99(5): 800-804.

23 Morlet N, Daniell M. Microbial keratitis: what's the preferred initial therapy? View 2: empirical fluoroquinolone therapy is sufficient initial treatment. Br J Ophthalmol. 2003; 87(9): 1169-1172.

24 Mezer E, Gelfand YA, Lotan R, Tamir A, Miller B. Bacteriological profile of ophthalmic infections in an Israeli hospital. Eur J Ophthalmol 1999; 9(2): 120-124.

25 Welby PL, Zusag TM, Storch GA. Comparison of the BACTEC Peds Plus pediatric blood culture vial with Roche pediatric Septi-Chek for blood cultures from pediatric patients. J Clin Microbiol 1992; 30(5): 1361-1362.

26 Hughes JG, Vetter EA, Patel R, Schleck CD, Harmsen S, Turgeant LT et al. Culture with BACTEC Peds Plus/F bottle compared with conventional methods for detection of bacteria in synovial fluid. J Clin Microbiol 2001; 39(12): 4468-4471.

27 Yagupsky P, Dagan R, Howard CW, Einhorn M, Kassis I, Simu A. High prevalence of Kingella kingae in joint fluid from children with septic arthritis revealed by the BACTEC blood culture system. J Clin Microbiol 1992; 30(5): 1278-1281.

28 Kratz A, Greenberg D, Barki Y, Cohen E, Lifshitz M. Pantoea agglomerans as a cause of septic arthritis after palm tree thorn injury; case report and literature review. Arch Dis Child 2003; 88(6): 542-544. 